\title{
Presentation of a mixed reality software with a HoloLens headset for a nutrition workshop
}

\author{
Hugo Rositi • Owen Kevin Appadoo • \\ Daniel Mestre • Sylvie Valarier . \\ Marie-Claire Ombret • Émilie \\ Gadea-Deschamps · Christine \\ Barret-Grimault • Christophe Lohou
}

November 24, 2020

\author{
H. Rositi \\ Université Clermont Auvergne, CNRS, SIGMA Clermont, Institut Pascal, F-63000 \\ Clermont-Ferrand, France \\ E-mail: hugo.rositi@uca.fr \\ Present address of H. Rositi: Université Clermont Auvergne, IUT de Clermont-Ferrand site \\ du Puy-en-Velay, Institut Pascal, 8 Rue Jean-Baptiste Fabre CS 1021943009 Le Puy-en- \\ Velay \\ ORCID: 0000-0002-5264-2027
}

O. K. Appadoo

Université Clermont Auvergne, CNRS, SIGMA Clermont, Institut Pascal, F-63000 Clermont-Ferrand, France

E-mail: owen.appadoo@uca.fr

D. Mestre

Aix-Marseille Université, CNRS, ISM Institut des Sciences du Mouvement - CP910, F-13288 Marseille, France

E-mail: daniel.mestre@univ-amu.fr

S. Valarier

Services de Chirurgie Bariatrique/Nutrition, C.H. Emile Roux du Puy-en-Velay, 12 boulevard Docteur Chantemesse, F-43000 Le Puy-en-Velay, France

E-mail: sylvie.valarier@ch-lepuy.fr

M.-C. Ombret

Unité transversale d'éducation du patient, C.H. Emile Roux du Puy-en-Velay, 12 boulevard Docteur Chantemesse, F-43000 Le Puy-en-Velay, France

E-mail: marieclaire.ombret@ch-lepuy.fr

É. Gadea-Deschamps

Unité de Recherche Clinique, C.H. Emile Roux du Puy-en-Velay, 12 boulevard Docteur Chantemesse, F-43000 Le Puy-en-Velay, France

E-mail: responsable.rechercheclinique@ch-lepuy.fr

C. Barret-Grimault

Services de Chirurgie Bariatrique/Nutrition, C.H. Emile Roux du Puy-en-Velay, 12 boulevard Docteur Chantemesse, F-43000 Le Puy-en-Velay, France

E-mail: christine.barret@ch-lepuy.fr

C. Lohou

Université Clermont Auvergne, CNRS, SIGMA Clermont, Institut Pascal, F-63000 
Abstract Microsoft has recently released a mixed reality headset called HoloLens. This semi-transparent visor headset allows the user who wears it to view the projection of $3 \mathrm{D}$ virtual objects placed in its real environment. The user can also grasp these 3D objects, which can also interact with each other. The framework of this new technology taking into account this physical approach (interactions, collisions) is called mixed reality. We had the opportunity to digitally transform a conventional nutrition workshop for patients waiting for bariatric surgery by designing a mixed reality software using the HoloLens headset. This software is called HOLO NUTRI.

In this paper, we present this software and its assessment (acceptance of this technology) by a cohort of thirty patients.

Keywords Mixed reality · HoloLens · therapeutic education · nutrition · clinical study

\section{Declarations}

- Funding: This study was funded by a french national grant PEPS INSIS CNRS.

- Conflicts of interests/Competing interests: The authors declare that they have no conflict of interest.

- Availability of data and material: Data concerning the clinical study are summarized through different tables in this paper.

- Code availability: Software is available on demand to C. Lohou.

- Authors' contributions: Hugo Rositi, Owen Kevin Appadoo and Christophe Lohou have contributed to the design, realisation and analysis of the clinical study; they have also contributed to the supervision of the software development and to the writing of this manuscript. Christine Barret-Grimault, Sylvie Valarier, Marie-Claire Ombret and Émilie GadeaDeschamps have contributed to the design, realisation and analysis of the clinical study. Daniel Mestre has contributed to the design thinking of the feedback forms.

\section{Context}

In this paper, we present a software application for patients waiting for bariatric surgery (modification of the anatomy of the digestive system). This application offers them to (virtually) compose their meal and then assess their choices; its educational objective is to inform patients about the modification of their diet (mainly, in terms of optimal quantities) after surgery.

This section is structured as follows: the terminology of targeted surgical procedures is presented in section 1.1; we then describe the conventional workshop in section 1.2 ; in section 1.3 , we list three main features expected in the

Clermont-Ferrand, France

E-mail: christophe.lohou@uca.fr 
application, one of which will fully justify the use of mixed reality; in section 1.4 , we will present the framework of mixed reality; in section 1.5 , we will review the state of the art of softwares used in nutritional therapeutic education; in section 1.6, we will present the software implemented for this mixed reality experience.

\subsection{Terminology}

Bariatric surgery aims at patient's weight loss. As part of our research, we are interested in the following two surgical solutions: reduction of the volume of the stomach "Sleeve Surgery" (Figure 1(a)), setting up a deviation from the normal path of progression of foods "Bypass Surgery" (Figure 1(b)).
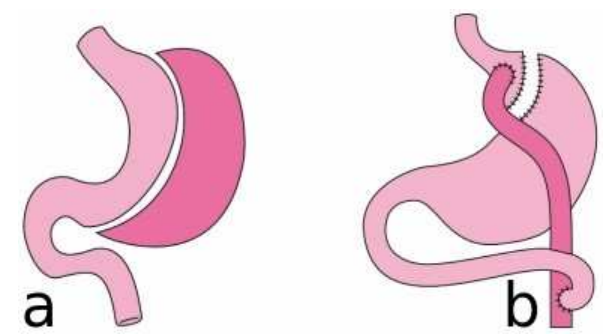

Fig. 1 Both types of considered interventions: (a) Sleeve Surgery - the dark part is removed, (b) Bypass surgery - the dark part represents the medium part of the small intestine that bypasses the normal food path. Figure from [1].

\subsection{Conventional workshop of nutritional therapeutic education}

The conventional nutritional therapeutic education workshop, later called CONV_WORK, is organized around the nine following steps (also summarized in Table 1 for a more global view): introduction of the session (Step 1); debate around the issue "To feed, eat, when one has been operated on a bariatric surgery, what does it evoke you ?" (Step 2); beginning of the workshop to discuss with patients about the daily diet to adopt after intervention (Step 3); patients are then invited to compose their menu by choosing printed cards representing different foods and in different quantities, the images come from the study SU.VI.MAX [2,3] (Step 4); collection by the clinical team of the menus developed by the patients (Step 5); collegial discussion on the choice made by each patient (Step 6); the dietician then gives to each patient advices according to the food and quantities that he or she chose (Step 7); dissemination of quantitative and qualitative messages (Step 8), qualitative messages focus on the need to vary the number of dishes, the expected duration of the meal, the importance of the chewing time, while quantitative messages focus 


\begin{tabular}{|l|l|}
\hline \multicolumn{1}{|c|}{ 1. Session introduction } \\
\hline 1.1. Presentation of the protocol and signature of the consent forms \\
\hline 1.2. Signature by patient \\
\hline 1.3. Group presentation (clinicians / patients) \\
\hline 1.4. Presentation of the objectives of the session \\
\hline $\begin{array}{l}\text { 2. Debate around the question "To feed, eat, when one has been operated on a bariatric } \\
\text { surgery, what does it evoke you ?" }\end{array}$ \\
\hline 3. Beginning of the workshop: Initial question "After the surgery, how to eat daily ?" \\
\hline \multicolumn{2}{|l|}{ Carrying out the workshop experience with cards } \\
\hline 4.1. Presentation of the workshop with cards \\
\hline 4.2. Food choices via cards \\
\hline 4.3. Register quantities in a table \\
\hline 5. Collection of the patients choices regarding the menus \\
\hline 6. Collegial discussion on the choice of each patient \\
\hline 7. Advices are given to the patient according to their food and quantities choices \\
\hline 8. Informative messages (quantitative and qualitative) \\
\hline 9. Forms (assessment knowledge + satisfaction) \\
\hline
\end{tabular}

Table 1 Description of the main constituent steps of the conventional workshop CONV_WORK

on reducing the amount of foods compared to a meal before surgery; finally, patients complete forms to evaluate the session (Step 9).

\subsection{Interests in digitalizing the conventional workshop}

We have proposed the digitalization of the classical workshop CONV_WORK, previously described in section 1.2. This new workshop, named DIGIT_ WORK afterwards, has the following main objectives to propose:

- the digital version of the menu composition using a software that should be rather playful. This software application is called HOLO_NUTRI,

- a more detailed analysis of the elaborated menu (several quantities associated by food, meal time estimation, taking into account of the chewing time, etc.), the numbers and quantities of foods being more important for this digitalized workshop make it possible to develop more personalized menus,

- an analog, in the computer application, of the patient's gesture when he or she takes one card representing a food and the associated quantity in the CONV_WORK workshop.

The first two items above can be achieved using the conventional computer/keyboard/screen/mouse set and software development. However, we have 
searched for the most appropriate solution for the implementation of the gesture to catch the digital model corresponding to the printed card of the food, or which would reinforce this learning [4]. To do this, we chose a solution setting up a mixed reality framework.

\subsection{Mixed reality}

To justify the framework of our study, we briefly recall the different virtual, augmented and mixed realities and the most commonly used hardware in such context.

In the context of virtual reality, for individual use, opaque headsets are the most used. The best known headsets are the HTC Vive [5] (Figure 2(a)) and Oculus Rift [6]. The main advantage of a virtual reality application is to be able, for example, to simulate an unrealistic environment (large-scale environments, etc.) and/or non real actors (avatars, etc.) and to provide immersive visualization (not related to the real environment). The main drawbacks are the discomfort associated with virtual reality headsets (cybersickness) [7], and a little high latency (time between the triggering of an action and the visual result related to this action). The main interactions by the wearer of this type of headset are carried out using handles (controllers) or gloves (Figure 2(a)). Other rather collective devices exist, such as CAVEs (Cave Automatic Virtual Environment) [8]. In brief, virtual reality headsets are built with opaque visor and prevent users to see their real environment; if taken into consideration, the content of the application can be handled by controllers.

With regard to augmented reality [9], the interest is to superimpose the visualization of $3 \mathrm{D}$ virtual objects to the one of the real environment. Most applications use markers to superimpose virtual objects (Figure 2(b))). The most common devices are smartphones, tablets for which the real environment is filmed using an embedded camera, or glasses (for example, Epson Moverio [10], Google Glass [11], etc.) and headsets for which the environment is perceived thanks to transparent glasses or to a semi-transparent visor $[12,13]$. The main downside of using tablets and smartphones is the need to hold them (Figure $2(\mathrm{c})$ ). If the application does not use markers, then it is necessary to use more sophisticated methods (such as registration for example), not necessarily exploitable or currently in development for light or inexpensive devices (or low quality cameras). This is why most of these devices (tablets, glasses, etc.) also deals mainly with 2D information (Figure 2(d)). Note also that until very recently, occlusion problems were not solved to take into account the real environment on smartphones [14], in other words, there is no real feeling of depth (Figure 2(e)). Interactions are performed using these same devices, or external cable-connected controllers.

Microsoft recently released the HoloLens headset [20]. In addition to scanning the real environment around the user (Figure 3(a)), this headset allows to display virtual $3 \mathrm{D}$ objects (called holograms) superimposed on the visualiza- 


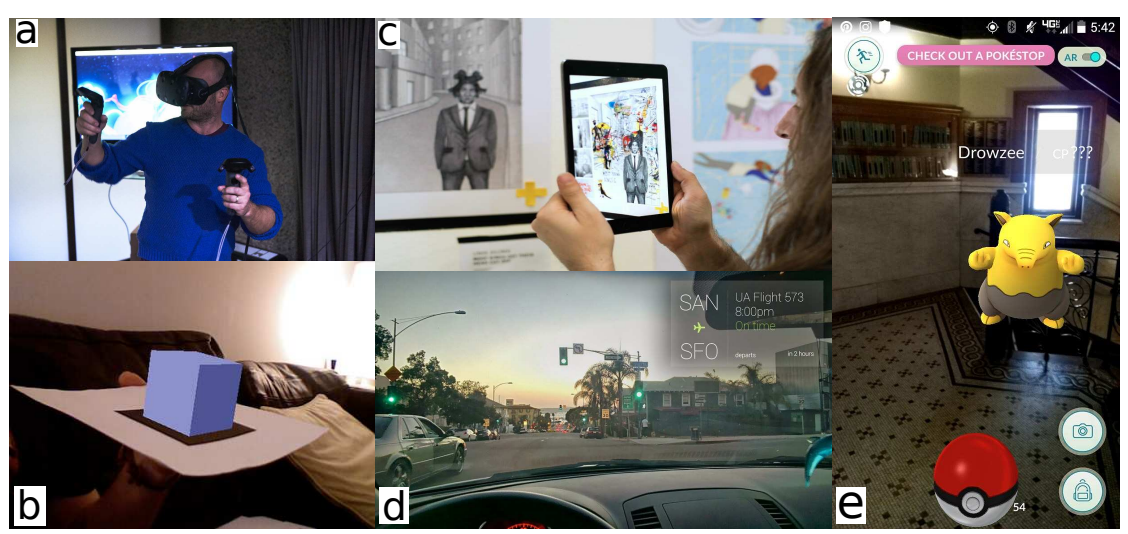

Fig. 2 (a) A user of the HTC Vive virtual reality headset, holding one controller in each hand. (b) Superimposition of a 3D virtual object on a marker. (c) Example of an augmented reality application using a tablet to scan a painting; there is a necessity to hold the device with one or two hands. (d) This view is from one user of the Google Glass device; the projected information are mainly in 2D and the user cannot interact with its environment. (e) The PokemonGO application on a mobile phone, occlusion problems persist and there is no interaction with the user environment. Subfigure (a) is from [15], subfigure (b) is from [16], subfigure (c) is from [17], subfigure (d) is from [18], subfigure (e) is from [19].

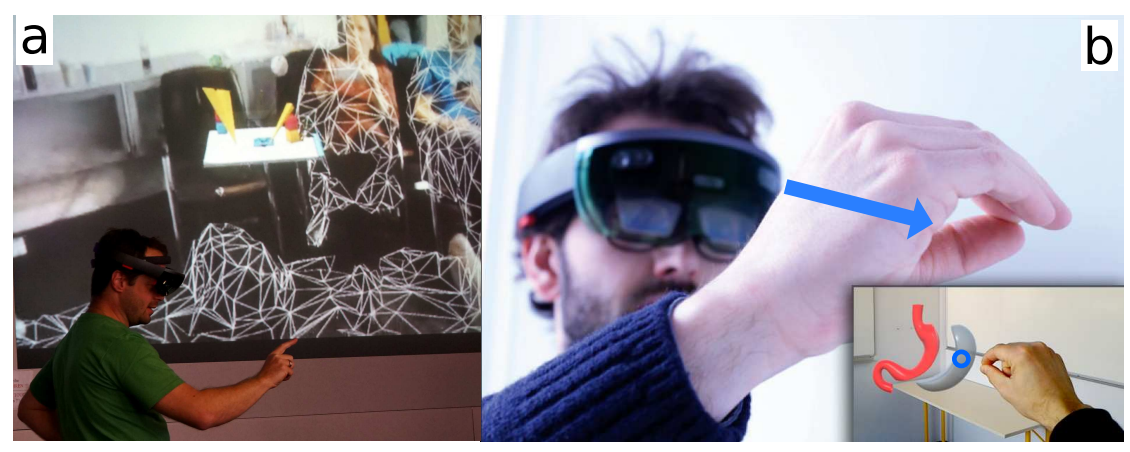

Fig. 3 (a) Example of the environment scanning performed by the HoloLens; it is decomposed in multiple triangles in order to create a 3D model of the scene where possible interactions will be allowed. (b) Photo montage illustrating the user of a HoloLens mixed reality headset and the content as seen by him in his own environment (boxed). The blue circle indicates the HoloLens cursor as seen by the user in the scene. The blue arrow indicates the gaze direction of the user. Subfigure (a) is from [21].

tion of the real environment, and to interact with these same objects thanks to the headset sensors (Figure 3(b)).

It is therefore the consideration of physics in order to manipulate holograms (collisions, etc.) that mainly distinguishes augmented reality from mixed reality. The user can interact with his environment using events from the Gaze, Gesture and Voice triplet (GGV) [22] by triggering actions programmed in the computer application to respond to these events. Indeed, the integrated gyroscope gives the movements of the head - Gaze, we highlight that this notion 
of gaze is defined by the orientation of the head (Figure 3(b)), the eyes are not tracked. Depth cameras of the headset allow it to follow some of the user's gestures (fingers pressed, hands opened, etc.) - Gesture and a microphone acquires the words or sounds emitted by the user - Voice.

Let us give more explanations about gaze interactions. When the user looks and keeps its gaze on an object (represented by the blue arrow on Figure 3(b)), if an interaction has been designed in the application with this object, it is therefore possible to interact with it; a blue cursor then appears on one of the 3D object's facet, the closest from the headset and in respect with the user gaze direction (thanks to the environment scanning (Figure 3(a))).

\subsection{State of the art of softwares in nutritional therapeutic education}

We will review some applications in relation to nutritional therapeutic education, according to the three frameworks of virtual, augmented and mixed realities.

\subsubsection{Virtual reality experiences}

Virtual reality experiences mainly consist of simulating meals or more specific situations (feeling of satiety, choice of sweet foods, etc.).

The simulation of meals may have more or less specific therapeutic aims. In [23], a meal simulation is proposed with, in addition to a virtual reality headset, an odour diffuser, a hearing device (sound of chewing) and a gripper (grasping the food), the objectives are to facilitate the management of nutritional disorders or to circumvent food allergies. Another meal simulation [24] uses electrical probe (salty sensation), thermal probe (spicy or menthol sensation) and electrode (chewing sensation) in addition to a virtual reality headset.

As more specific situations, a serious game has been proposed in [25] with a virtual reality headset to take into account the differences in human body reactions depending on sugar consumption (see also [26]). We can list other works, for example, modifying the visualization, inside the headset, of the volume of food ingested in order to accelerate the impression of being satiated [27]; or the simulation with a focus on food selection [28]; or the modification of the body-shape [29]. In [30], the validation of the use of virtual reality is questioned for assessing parents' child feeding behavior. Another research work will be noted (neuroscience framework) to decouple the desire to eat from the "intrinsic survival" notion (feeding) [31]. Other experiments using the term virtual reality are in fact carried out without immersion: for the treatment of obesity in an individual way in front of a screen [32], or even a shopping experience (food) in a 3D supermarket [33]. 


\subsubsection{Experiences in augmented reality}

Augmented reality experiments mainly consist in providing nutritional information on food products either punctually and individually, or in the form of a medical follow-up.

We can list projects to get information about the composition of meals, with scanners (camera sensors of smartphones and/or tablets) to visualize nutritional information [34], for the recognition of a fruit and its composition [35], to provide a nutritional response according to a predefined diet (preferences or intolerances) [36] or more specifically for sweet drinks [37]. Other hardware also provide access to this information such as connected watches in [38] which use information on food packaging by RFID (Radio Frequency IDentification) tags. In [39], a system of aid for the purchase of food products in a supermarket is proposed based on individual or nutritional criteria. Finally, other projects aimed at nutritional education in general are presented in the reference [40], such as managing children's appetite for vegetables.

Regarding the medical follow-up, work has been proposed for different categories of people (diabetic patients, pregnant women, etc.): analysis of the management of the care for diabetic patients by mobile applications [41,42], follow-up of meals taken for several days by pregnant women [43], estimation of delivered portions $[44,45]$.

\subsubsection{Mixed reality experiences}

The HoloLens headset is used in the medical context mainly for two types of applications: teaching $[46,47,48]$ or visual assistance in surgery (arterial network [49], prostate [50], shoulder [51]). To our knowledge, only the reference [52] (nutritional diseases - overweight management) makes a link between HoloLens and the context related to our work.

In the experience described in this paper, as previously stated, the main objective of our application is to compose a meal while strengthening the choice of foods. In order to do that, we have chosen to provide a gesture learning reinforcement which justifies the mixed reality framework. In addition, qualitative and quantitative messages are not focused on the nutritional composition of each food (unlike nutritional scanners) but on the composition of the entire meal. Here, the patient-specific follow-up is limited to verify that the qualitative and quantitative messages have been retained at different times after this experiment.

\subsection{Technological justification}

It is therefore this mixed reality technology using the HoloLens headset that we have chosen for the digital transformation of the CONV WORK workshop for the following reasons: 
- patients continue to see the real environment around them through the semi-transparent visor, this avoids any additional stress that can occur with opaque virtual reality headsets (Figure 3(b)),

- patients must wear this headset about thirty minutes in a one-and-a-half hour session. This seems to us to be too long for uninitiated users of virtual reality. We also have assumed that mixed reality would reinforce learning in the DIGIT_WORK digital workshop. In fact, unlike virtual reality headsets, here, the user acts and sees the movement of his arm, the action of catching a food with the hand. Unlike phones and tablets using at least one hand of the user or the one of an assistant (augmented reality framework), here the user can perform the gesture freely with the HoloLens headset on her/his head (yet the gesture has to be done in an area defined by the headset sensors) (Figure 3(b)).

\subsection{Plan of the article}

In Section 2, we present the developed application and its features. Section 3 presents the framework in which this application was used and the results of the evaluation of its use by a cohort of thirty patients. In Section 4, we will discuss the first conclusions of this study.

\section{Application}

\subsection{List of expected features}

The specifications of our application is composed of two parts. The first part must allow the patient to clearly understand the type of operation he or she will undergo (by visualizing the stomach before and after surgery). The second part must provide an interactive composition of a standard menu in two iterations:

- the first iteration gives little guidance to the patient and displays at the end a first analysis (qualitative and quantitative messages),

- the second iteration displays more information (visualization of the stomach in transparency showing its filling as the food is ingested, display of the number of portions), which should allow the patient to better compose his menu.

During both parts of the application, it is requested that the user is the most active possible: for the first part, the user must remove a portion of the stomach depending on the envisaged intervention; for the second part, the patient must compose his meal by choosing foods, one after the other, and their ingestion will then be simulated by placing each food in a "virtual" jaw, which will trigger a chewing animation and prevent the patient from selecting a new food until the chewing time has not elapsed.

We have chosen to treat this type of interaction with mixed reality in order to have a maximum impact on the patient (learning by gesture): it is with the 
hands that he or she will interact to remove a part of the stomach (first part), and also to choose foods when placing them into the virtual jaw (second part).

\subsection{Development environment}

Whatever the type of reality envisaged, the development of computer applications to use this hardware is done either from proprietary SDKs (Software Development Kit) or by using interactive $3 \mathrm{D}$ graphical application development engines such as Unity [53] and Unreal Engine [54], originally designed for the development of video games but now also addressed to wider professional sectors (automotive, AEC -Architectural, Engineering and Construction-, etc.).

For our application, we first design several 3D resources $(3 \mathrm{D}$ models and animations made in Blender software [55], see Section 2.3). The development was carried out with the Unity engine [53] (import of 2D images, 3D models and animations). Several scripts were designed to describe the wanted user interactions on 3D objects; these scripts (integrated in our Unity framework) were written with the $\mathrm{C} \#$ language from the Microsoft Visual Studio programming environment [56] and with the HoloToolKit library [57] specialized for mixed reality with the HoloLens headset. Once all these assets are compiled, it produces our software HOLO _ NUTRI (that can be tested on a PC through an emulator). By plugging the HoloLens headset into the $\mathrm{PC}$, the application can then be deployed/embedded into the headset. The user, wearing the headset, can then run HOLO_NUTRI from the HoloLens main menu without the headset being connected to a PC (by either cable or WiFi).

\subsection{Digital contents}

The different 3D models (stomach and jaw) were made with the software Blender [55], these models must not have too many polygons to maintain the smooth running of the software [58]. For the food selection stage, these are represented by $2 \mathrm{D}$ images from SU.VI.MAX study $[2,3]$. Once the food is selected, it appears as a cube, which will be easier for the patient to handle than a $2 \mathrm{D}$ image. The size of this cube is based on the quantity selected for the selected food.

\subsection{Description of the application HOLO_NUTRI}

\subsubsection{First part: basic simulation of the surgical intervention}

The first part proposes to the user to choose the type of intervention that he or she will undergo (either using buttons in the application (Figure 4(a)), or by scanning a leaflet with the HoloLens (Figure 4(b)), knowing that there is one leaflet for each type of intervention. 


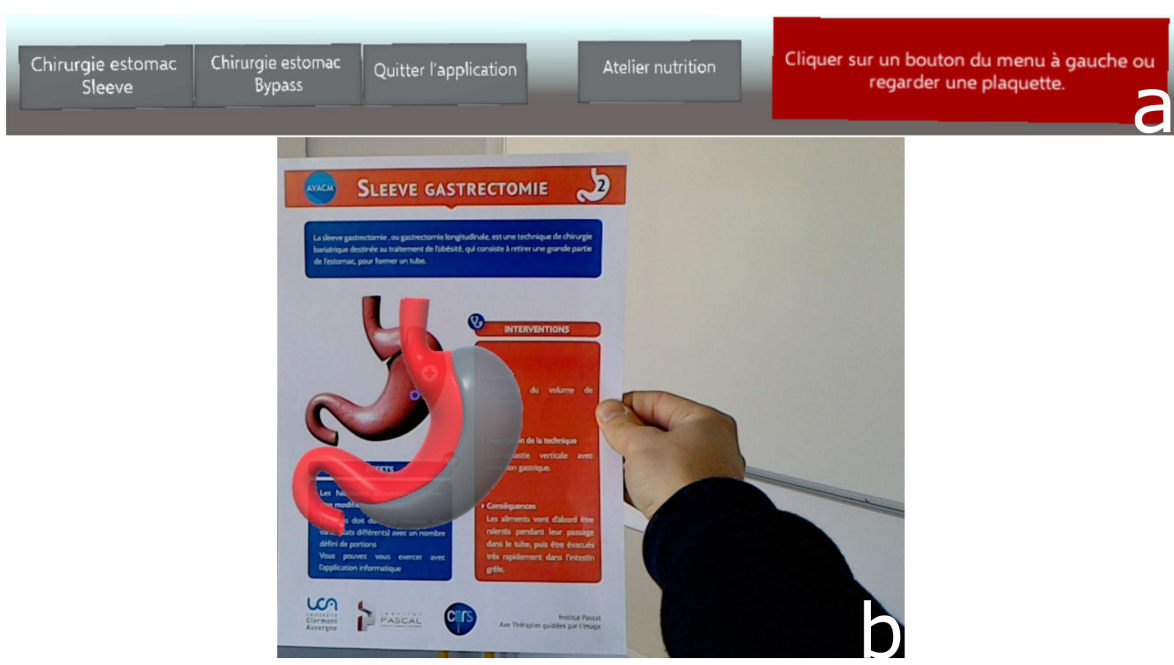

Fig. 4 (a) "Sleeve Surgery" button (first button on the left in the upper menu) and "Bypass" button (second bouton on the left in the upper menu). (b) Scanned leaflet with 3D model (Sleeve surgery).

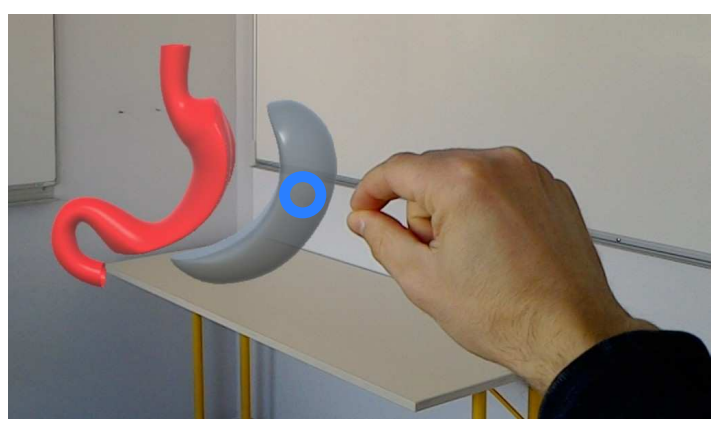

Fig. 5 User interaction with the two parts (red and gray) of the virtual stomach during the second step of HOLO_NUTRI. The user virtually picks the gray shape of the stomach to take it away from the main red shape that represents the retained stomach part during surgery. From the user point of view, its hand and the object to be moved are aligned (photo taken from the HoloLens camera induces a misalignment).

Then a virtual stomach is displayed and the user will interact with it (Figure 5). For example in the sleeve operation (Section 1.1 and Figure 1(a)) the user, by pinching its thumb and forefinger, will select a part of the stomach that is supposed to be removed by moving it aside from the main part of the stomach. This part was not present in the conventional workshop CONV_WORK. Once this action is done, the second part of HOLO_NUTRI starts.

\subsubsection{Second part: Meal composition}

The second part displays a virtual buffet of about thirty different foods. Due to the quantity of informations and in order to render those informations in 
the most visible way, this buffet is provided in a semi vertical cylinder manner, with for the same column, three different portion sizes (small, medium, large) of the same food (Figures 6 and 7 ).

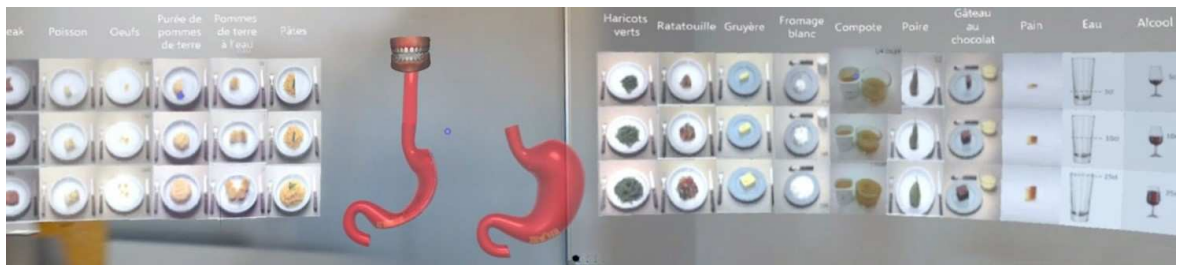

Fig. 6 Buffet screenshot (part of the semi cylinder).

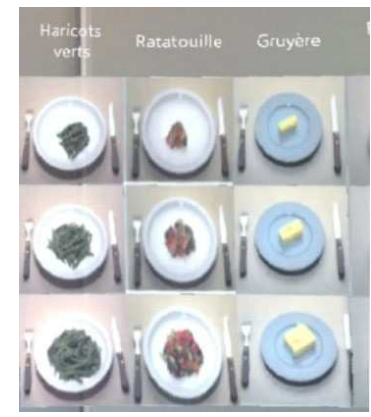

Fig. 7 Different portions for the same food.

The user chooses the food by looking at it through the transparent visor (HoloLens' gaze) then the user can grab it by pinching his/her fingers together and by releasing them afterwards (HoloLens' gesture). The selected food appears as a cube in front of the 3D jaw (Figure 8(a)). The user grabs once again the food and brings it to his/her own mouth, an animation is then triggered (opening and closing cycle of the jaw, Figures 8(a) and (b)), forbidding any other action from the user, this essential step is designed to draw attention on the importance of the chewing time. The user repeats this procedure as long as he or she believes it is necessary to add foods to fulfill her/his meal. When the user is done, a first recap is shown to inform about the number of food selected and the portion size associated (Figure 9). In a next step, qualitiative (Figure 10(a)) and quantitative (Figure 10(b)) messages are displayed (according to the ones delivered in the conventional workshop). The qualitative messages inform on the necessity to have a diet as various as possible and to observe the chewing time (Figure 10(a)). The quantitative messages point the significant stomach volume reduction out (Figure 10(b)). The user is then allowed to perform a second iteration of this part of the workshop. For this second iteration, supplementary indicators (number of swallowed foods (Figure 11), 
transparent stomach (Figure 12), etc.) may help her or him to better compose their meals.

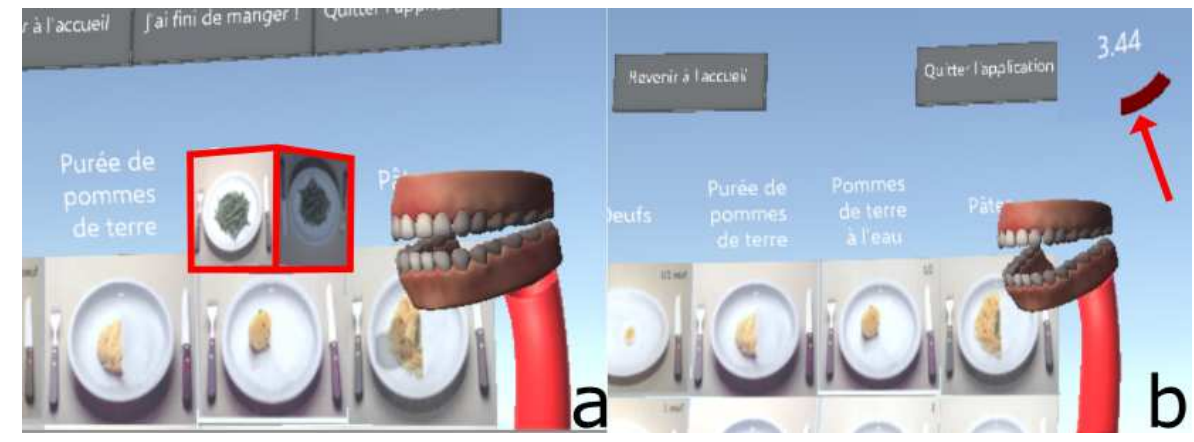

Fig. 8 (a) 3D representation of foods (red delineated cube) and of the jawbone. (b) Jawbone animation (opening-closing cycle) and display of the remaining chewing time (red arrow).

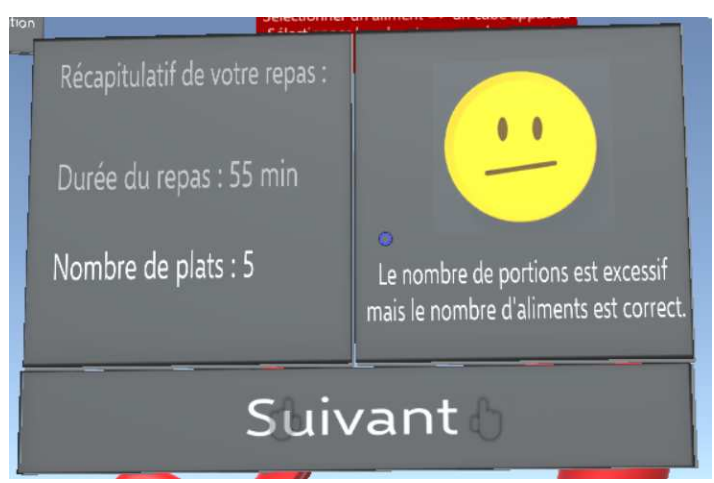

Fig. 9 Recap panel of the meal; on the left are shown the meal duration and the number of selected foods, on the right a message about the number of portions and foods composing the meal and a logo corresponding to that message. 


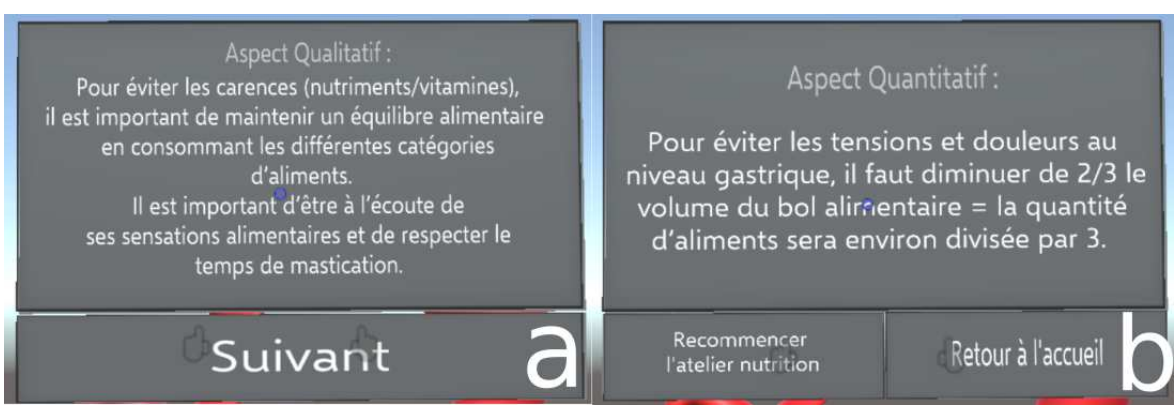

Fig. 10 Informative panels on the qualitative (a) and quantitative (b) aspects of the meal.

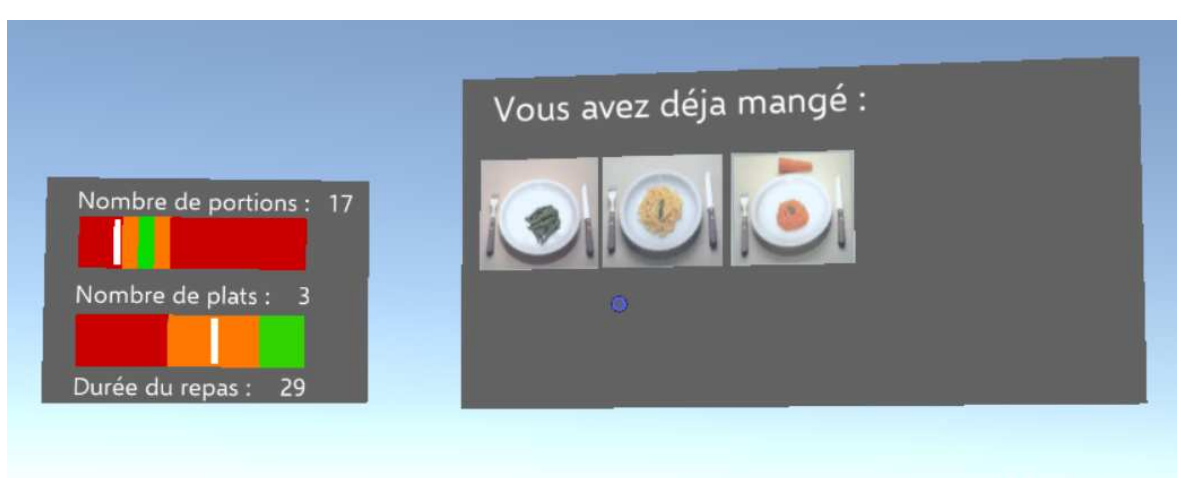

Fig. 11 Supplementary indicators for the second iteration: on the left, portions and foods numbers along with the duration of the meal are displayed; on the right, swallowed foods are shown.

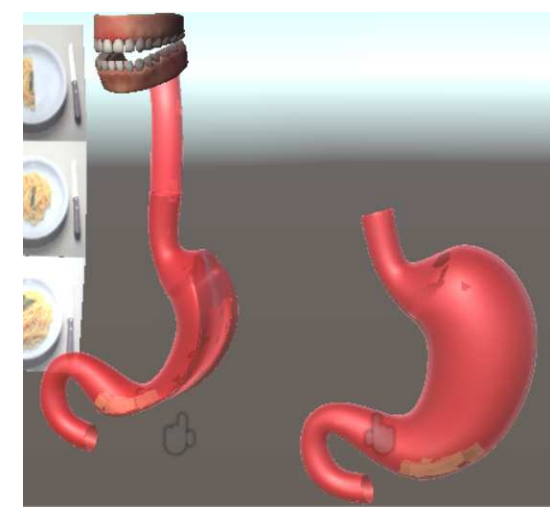

Fig. 12 Transparency of the 3D stomachs (on the right, before surgery and on the left, after surgery) to enhance visualization of the occupied volume into them by the swallowed foods. 


\section{Results}

\subsection{Digital workshop presentation}

HOLO_NUTRI software has been used by patients during the DIGIT _ WORK workshop which goal was to make patients aware of the post-surgery diet modification they will endure due to the bariatric surgery they will undergo. This workshop is composed of nine steps (also depicted in Table 2 for a more global understanding): session introduction (step 1), identic to step 1 of CONV_WORK workshop; debate around the central question "To feed, eat, when one has been operated on a bariatric surgery, what does it evoke you ?" (step 2), identic to step 2 of CONV_ WORK workshop; computer scientists team presentation (step 3); hardware and its using presentation (step 4) by showing a video. A dedicated software named LEARN_EX has been developped by us to introduce basic gestures to control the HoloLens and essential to the good use of HOLO_NUTRI (Figure 13). The computer scientist team gives information about this LEARN_EX learning exercise (step 5). This workshop starts with a discussion between all patients about the daily diet post-surgery (step 6), identic to step 5 of CONV_ WORK workshop; patients are then invited to compose their own meal with HOLO_NUTRI software (step 7); the nutritionist delivers several advices depending on the food and quantities selected by the patient (step 8); feedback forms are then filled by patients in order to evaluate the session (step 9), identic to step 9 of CONV WORK workshop. We have set up a specific communication to make patients able to use this technology and application as fast as possible: this communication is composed of laminated leaflets (surgery details, gestures to be used with the headset), several video sequences (basic gestures description to use both the headset and the application), and also a custom-made learning exercise LEARN_EX (to address specific gestures). More details about this dedicated communication is available in [1].
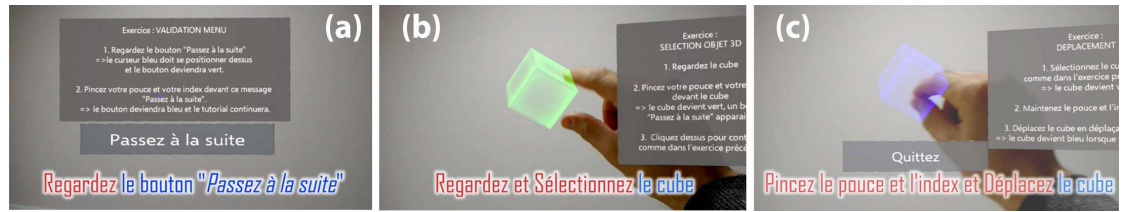

Fig. 13 The consecutive three steps of LEARN_EX learning exercise : (a) Menu validation, (b) $3 \mathrm{D}$ object selection, (c) $3 \mathrm{D}$ object movement.

\subsection{Experimental procedure}

The software presented in this article has been used in a hospital hosted workshop that aimed to help patient prepare for a bariatric surgery (Centre Hospi- 


\begin{tabular}{|c|c|}
\hline & Session introduction \\
\hline & 1.1. Experimental protocol presentation and free consent form signature \\
\hline & 1.2. Signature of the free consent form by each patient \\
\hline & 1.3. Group presentation (clinicians/patients) \\
\hline & 1.4. Session objectives presentation \\
\hline & $\begin{array}{l}\text { Debate around the question "To feed, eat, when one has been operated on a bariatric } \\
\text { surgery, what does it evoke you ?" }\end{array}$ \\
\hline & Group presentation (computer scientists/patients) \\
\hline & Material presentation \\
\hline & 4.1. HoloLens presentation \\
\hline & 4.2. Headset presentation and its carrying (video sequence) \\
\hline & 4.3. Gesture presentation (video sequence) \\
\hline & 4.4. Reminder leaflet delivery \\
\hline & Learning exercise \\
\hline & 5.1. Learning exercise presentation (video sequence) \\
\hline & 5.2. Reminder leaflet delivery \\
\hline & 5.3. Headset setup (computer scientists/patients) \\
\hline & 5.4. Launching of learning exercise LEARN EX \\
\hline & 5.5. Headset removal \\
\hline & Workshop trigger \\
\hline & 6.1. Starting question «After the surgery, how to eat daily ?») \\
\hline & HOLO NUTRI application \\
\hline & 7.1. Presentation of HOLO NUTRI application (video sequence) \\
\hline & 7.2. Distribution of a paper leaflet reminder on the application \\
\hline & 7.3. Headset setup (computer scientists/patients) \\
\hline & 7.4. Launching of HOLO NUTRI \\
\hline & 7.4.1. Surgical operation simulation (using leaflet or the menu buttons) \\
\hline & 7.4.2. First iteration (simple interface) \\
\hline & 7.4.3. Food choice via $2 \mathrm{D}$ images in the virtual self-service restaurant \\
\hline & 7.4.4. Recap panel, analyse of patient choices and informative messages \\
\hline & $\begin{array}{l}\text { 7.4.5. Second iteration (same interface with more visual information and with } \\
\text { continuous analysis of the food choice) }\end{array}$ \\
\hline & 7.5 Headset removal \\
\hline & . Informative messages (quantitative and qualitative) \\
\hline & \\
\hline & Forms (assessment knowledge + satisfaction) \\
\hline
\end{tabular}

Table 2 Description of the main constituent steps of the digital workshop DIGIT WORK.

talier Émile Roux, Le Puy-en-Velay, France), several sessions of one hour and a half had been organized. For each one of them, a group of three patients were supposed to simultaneously handle first the headset HoloLens then to use the application HOLO_NUTRI. We, on purpose, limited the number of participants to three for the following reasons: size of the experimental room (Figure 14), only three HoloLens at our disposal, involvement of three researchers to help patients in the use of both the equipment and the software. 
To allow this simultaneous participation of three patients, we have used the HoloLens App application [59] from the Microsoft Store in order to connect (using WiFi) the three HoloLens to three different computers to get access to a video feedback of each patient to better help them in their use of both this hardware and the software (Figure 14).

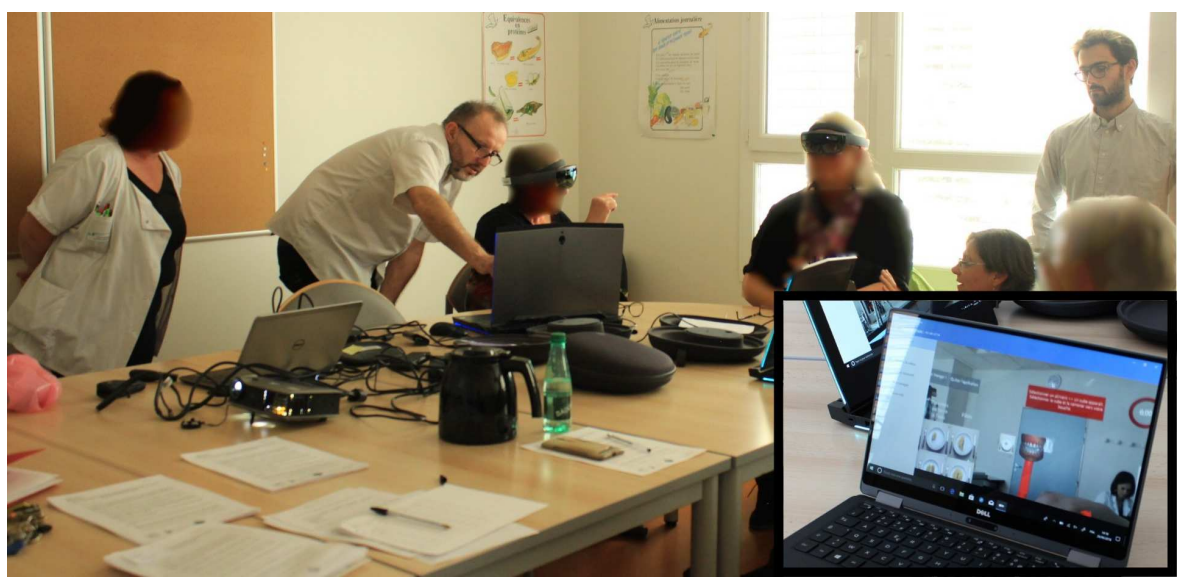

Fig. 14 DIGIT WORK workshop layout into the experimental room. In the box, video feedback of HOLO_NUTRI on a laptop.

\subsection{Application evaluation by the patients}

HOLO_NUTRI has been used and assessed for 10 sessions by a total of 30 patients ( 27 women and 3 men) evenly distributed among those 10 sessions. These questions (12 in number) have been gathered into five different categories; the answers to these questions are shown in Tables 3 to 7 . A number of these questions can be answered by a simple yes or no, while others expect an answer by scale (e.g. not at all; slightly; moderately; severely). We write in each table the number of patients answering a given answer (by column) in the first line and the corresponding percentage in the second line in regard to the whole cohort of 30 patients. The average age of this group is 46 years old (standard deviation of 11.57 years, with a minimum age of 21 and a maximum age of 68). The average duration of the workshop introduction (explanations and video presentation of the headset) and of both the presentation and the completion of LEARN_EX is 13:07 minutes (standard deviation of 2:32 minutes, minimal duration of 8 minutes and maximal of 22 minutes). The mean duration use of HOLO_NUTRI simulation (first part) and two iterations of the meal composition (second part) is 17:53 minutes (standard deviation of 4:05 minutes, minimal duration of 12 minutes and maximal duration of 30 


\begin{tabular}{|l|c|c|}
\hline & Yes & No \\
\hline Q1. Have you ever used an augmented reality material or application & 6 & 24 \\
\cline { 2 - 3 } before (e.g. Pokemon Go, Snapchat, etc.)? & $20 \%$ & $80 \%$ \\
\hline
\end{tabular}

Table 3 Evaluation of patient knowledge on augmented reality devices and related applications.

\begin{tabular}{|l|c|c|c|c|c|}
\hline $\begin{array}{l}\text { Q2. Have you felt any discom- } \\
\text { fort due to wearing } \\
\text { the headset: }\end{array}$ & Not & Slightly & Moder- & Sever- & Missing \\
\hline \multirow{2}{*}{ a. General discomfort ? } & 15 & 11 & 3 & 1 & data \\
\hline \multirow{2}{*}{ b. General tiredness ? } & $50 \%$ & $36.67 \%$ & $10 \%$ & $3.33 \%$ & \\
\hline \multirow{2}{*}{ c. Ocular tiredness ? } & 27 & 1 & 1 & 0 & 1 \\
\hline \multirow{2}{*}{ d. Nauseas ? } & $90 \%$ & $3.33 \%$ & $3.33 \%$ & $0 \%$ & $3.33 \%$ \\
\cline { 2 - 6 } & $46.67 \%$ & 11 & 4 & 1 & \\
\cline { 2 - 6 } & 29 & 0 & 1 & 0 & \\
\hline
\end{tabular}

Table 4 Evaluation of patient physical feeling during the experiment.

minutes). For five of those patients, the duration of the different workshop phases could not have been recorded.

We have provided a series of questions to patients in order to get their feedback about this mixed reality experience. Those questions were focused on the patient technological knowledge (Table 3, Q1), on the physical feeling during the experience (Table 4, Q2), on the assessment of the software use (Table 5, Q3-Q7), on the assessment of this mixed reality technology compared to a more conventional one with the set computer/keyboard/screen/mouse (Table 6, Q8-Q11) and on the expected educational benefit (Table 7, Q12).

We present these five groups of results in the following tables from 3 to 7 .

- (Group 1, Table 3). $80 \%$ of patients state that they have no technological skill at all in the use of augmented reality material or application. We can then conclude that this experience is the first with this kind of mixed reality material for most of them.

- (Group 2, Table 4). Half of our patient cohort does not seem to feel any general discomfort (Q2a). Only 2 patients among 29 (1 missing data) state feeling general tiredness following the experience (Q2b). 16 patients declare feeling a slight to severe ocular tiredness (Q2c). Only one patient has felt nauseous (Q2d). Over the 30 patients, 3 have found the headset to be heavy, 2 showed headaches with 1 who has declared feeling a severe ocular tiredness (Q2c). We can conclude that the physical feeling of patients is rather satisfying considering that this experience is the first with mixed reality for most of them.

- (Group 3, Table 5). 28 patients report not feeling the workload to be too much tiring (Q3). 24 patients have felt rather relaxed, satisfied and selfconfident while using the HOLO NUTRI software (Q4). The duration of this experience has been perceived as rather satisfying by 28 patients (as a reminder, the session was planned to last one hour and a half with an effective use of HOLO_NUTRI of 18 minutes) (Q5), the two patients who have not 


\begin{tabular}{|l|c|c|c|c|}
\hline & $\begin{array}{c}\text { Not } \\
\text { at all }\end{array}$ & $\begin{array}{c}\text { Not } \\
\text { really }\end{array}$ & $\begin{array}{c}\text { Yes } \\
\text { rather }\end{array}$ & $\begin{array}{c}\text { Yes } \\
\text { absolutely }\end{array}$ \\
\hline $\begin{array}{l}\text { Q3. Do you find this experiment workload } \\
\text { tiring? }\end{array}$ & 18 & 10 & 2 & 0 \\
\cline { 2 - 5 } & $60 \%$ & $33.33 \%$ & $6.67 \%$ & $0 \%$ \\
\hline \multirow{2}{*}{$\begin{array}{l}\text { Q4. During this nutritional workshop, did } \\
\text { you feel satisfied, relaxed, self-confident ? }\end{array}$} & 0 & 6 & 19 & 5 \\
\cline { 2 - 5 } & $0 \%$ & $20 \%$ & $63.33 \%$ & $16.67 \%$ \\
\hline $\begin{array}{l}\text { Q5. Was the duration of this experience } \\
\text { satisfying? }\end{array}$ & 0 & 2 & 20 & 8 \\
\hline $\begin{array}{l}\text { Q6. Would you recommend this experience } \\
\text { to other patients? }\end{array}$ & 0 & 2 & 13 & 15 \\
\hline \multirow{2}{*}{$\begin{array}{l}\text { Q7. From a general point of view, how do } \\
\text { you evaluate this experience ? }\end{array}$} & 0 & 3 & 18 & $50 \%$ \\
\cline { 2 - 5 } & $0 \%$ & $10 \%$ & $60 \%$ & $30 \%$ \\
\hline
\end{tabular}

Table 5 Assessment of HOLO_NUTRI application use by the patients.

appreciated the duration have also declared feeling ocular tiredness (Table 4, Q2c). 28 patients would recommend this experience and 15 of them with no restriction (Q6); 2 patients would not really recommend this experience, one finds no interest in this workshop compared to the classic CONV_WORK and the other finds this technology too modern, requiring a very high level of concentration to use this device which deviates attention from the main goal of the workshop, this patient has also declared feeling general tiredness following this experience (Table 4, Q2b). Three patients only have not perceived this experience as satisfying (Q7): two of them for the reason previously stated in the question about recommendation to another person (Q6), and the last patient has not given us any feedback. In a summary, this experience has been rather tough for three patients (difficulties in performing the required gestures willing to a certain annoyance and preventing them from fulfilling the workshop goals, i.e. the total completion of two iterations of the second part of HOLO_NUTRI). For the rest of the cohort, this experience has been perceived as satisfying to very satisfying, which is rather positive to repeat this kind of mixed reality experience in a medical context, despite technologies that could be seen as too modern and not user-friendly. The same bias seem to be refuted by the results we present on the assessment of this new technology over a more conventional approach (Table 4 ).

- (Group 4, Table 6). 28 patients have not been too much disturbed by the superimposition of virtual elements onto their real environment (Q8). To our great surprise, a majority of patients has believed to handle real objects (14 "yes absolutely" and 4 "yes rather") during the use of HOLO_NUTRI (Q9). About the patient's focus on the workshop goal, more than $75 \%$ of them stated not being troubled by the use of this technology (17 "not at all" and 8 "not really"); 5 patients have stated to feel a focus trouble due to the use of this material (Q10). Finally, $60 \%$ of the patient cohort declared to be rather interested in having a workshop with this kind of material and set up rather than with a more conventional approach (the set computer/keyboard/screen/mouse or 


\begin{tabular}{|c|c|c|c|c|}
\hline & $\begin{array}{l}\text { Not } \\
\text { at all }\end{array}$ & $\begin{array}{l}\text { Not } \\
\text { really }\end{array}$ & $\begin{array}{c}\text { Yes } \\
\text { rather }\end{array}$ & $\begin{array}{c}\text { Yes } \\
\text { absolutely }\end{array}$ \\
\hline \multirow{2}{*}{$\begin{array}{l}\text { Q8. Does the fact to see at the same time a } \\
\text { real and virtual environment disturbed you? }\end{array}$} & 18 & 10 & 2 & 0 \\
\hline & $60 \%$ & $33.33 \%$ & $6.67 \%$ & $0 \%$ \\
\hline \multirow{2}{*}{$\begin{array}{l}\text { Q9. During the use of this application, did } \\
\text { you believe you were handling real objects ? }\end{array}$} & 2 & 10 & 14 & 4 \\
\hline & $6.67 \%$ & $33.33 \%$ & $46.67 \%$ & $13.33 \%$ \\
\hline \multirow{2}{*}{$\begin{array}{l}\text { Q10. Did this technological device prevent } \\
\text { you from focusing on the goals of this } \\
\text { workshop? }\end{array}$} & 17 & 8 & 5 & 0 \\
\hline & $56.67 \%$ & $26.67 \%$ & $16.67 \%$ & $0 \%$ \\
\hline \multirow{2}{*}{$\begin{array}{l}\text { Q11. Would you better prefer to use a } \\
\text { computer or a tablet with mouse-like gestures } \\
\text { (2D) compared to this experience? }\end{array}$} & 8 & 10 & 8 & 4 \\
\hline & $26.67 \%$ & $33.33 \%$ & $26.67 \%$ & $13.33 \%$ \\
\hline
\end{tabular}

Table 6 Assessment by the patients of this mixed reality technology compared to a more conventional information technology approach (computer/keyboard/screen/mouse).

\begin{tabular}{|l|c|c|c|c|c|}
\hline & $\begin{array}{c}\text { Not } \\
\text { at all }\end{array}$ & $\begin{array}{c}\text { Not } \\
\text { really }\end{array}$ & $\begin{array}{c}\text { Yes } \\
\text { rather }\end{array}$ & $\begin{array}{c}\text { Yes } \\
\text { absolutely }\end{array}$ & $\begin{array}{c}\text { Missing } \\
\text { data }\end{array}$ \\
\hline $\begin{array}{l}\text { Q12. Do you think that the } \\
\text { possibility to handle virtual } \\
\text { objects make the learning more } \\
\text { attractive and interesting? }\end{array}$ & 0 & 1 & 17 & 11 & 1 \\
\cline { 2 - 6 } & $0 \%$ & $3.33 \%$ & $56.67 \%$ & $36.67 \%$ & $3.33 \%$ \\
\hline
\end{tabular}

Table 7 Expected educational benefit evaluation from the patient's point of view.

tablet) (Q11). The summary of the use of mixed reality in this awareness workshop on post bariatric surgery nutrition is, in accordance with the feedback we get from the patient themselves, rather positive and quite well perceived; this experience has been rather well received.

- (Group 5, Table 7). A question relative to the digitalization of this workshop is to understand if we can expect a better educational benefit with a mixed reality headset and an associated application compared to a classic information technology approach (computer/keyboard/screen/mouse set). We briefly speak about this question in this communication but it is presented with more detailed information in [4]. Thus, the majority of the patient cohort that represents $93 \%$ (excepting one patient and one missing data to this question) has found a real motivation in learning from the nutritional messages delivered through the handling of virtual objects (i.e. meal composition with 3D foods).

\section{Discussion and conclusion}

We will start by listing the positive and negative aspects we have noted during this mixed reality experience in a medical context. We will consider five 
criteria: 1. material, 2. software, 3. developer, 4. clinical, 5. patient. We will conclude this section by a discussion and by giving perspectives to this application and its framework.

\section{Positive aspects:}

1. The material we used is working in an autonomous way, an embedded computer is stored in the headset itself. No other equipment is needed to work, all sensors are present in or on the headset; no cable connexion is required.

2. With an appropriate communication (e.g. video tutorial), 20 minutes are enough to handle this equipment and the application [1].

3. Application development for the HoloLens may benefit from several frameworks dedicated to development of 3D interactive graphical applications such as Unity and Vuforia. Therefore, there is no need to program with a low level library like DirectX [58]

4. More and more medical applications use the HoloLens headset. We can note two main uses:

- an only educative use (e.g. the headset may work as a camera to broadcast a surgeon point of view to an audience of students in order to provide a better understanding of the surgical gestures to perform),

- during a surgical operation, a visualization of the pre-operative data is given to the surgeon (e.g. patient clinical file, patient 3D organ models, etc.); this may represent a real help for the surgeon in its practice $[49$, $60,61,62,63]$. This visualization is mainly provided without any registration.

5. This new technology allows researchers to address new problems, by bringing efficient and innovative answer like in our experience we can note a reinforced learning from the gesture [4] and therefore bring a real benefit to the patient in the end.

\section{Negative aspects:}

1. This material carries some limitations such as its high financial cost, a low screen resolution, a narrow field of view and a quite heavy weight.

2. The release of HoloLens 2 less than two years and a half after the first version in France, asks us the necessity to update the HOLO_NUTRI application.

3. For the developer, the learning curve is steep because it needs Unity knowledge, several in-development libraries and the dedicated documentation, which is not keep up-to-date on a regular basis. A specific development is required to take in consideration the specificity of each environment where the application will be used. For us, a specific distance was tuned to allow patients to easily grab the virtual 3D food, size of the semi-cylinder was also tuned to fit with the HoloLens field-of-view [58].

4. Non computer scientist members of this experience would not feel comfortable enough with this kind of material to perform this whole experience in an autonomous way. 
5. From our side, it was not possible to let this kind of material at the free disposal of the patients, therefore, we could not consider to perform this experience at home (technical difficulties to handle this material without assistance) and even less a long term follow-up; unlike augmented reality based on the use of a sole smartphone (as described in section 1.5), which would give more autonomy to the patients.

To conclude, we realised a translational research study (from the clinical needs towards the patient) with limited means (one student, three months of development, one Unity licence and one computer for programming), not forgetting the purchase of the HoloLens. In the frame of this nutritional workshop, we remind that $60 \%$ of patients who participated in this experience have assessed that this mixed reality approach was preferable to a more conventional one (computer/keyboard/screen/mouse set). Thus, we can easily extend this kind of work to other pathologies or to similar prevention workshops. We would like to insist on the fact that it would have been really hard to consider the same application without the HoloLens material. The use of 3D models in our application does not limit to the sole visualization, to a spatial positioning in a virtual environment or to a scaling of those latest but it tends to make the patient the more active as possible by giving them the opportunity to handle them directly. This gesture reinforced learning is reviewed in [4]. Our future work would be to add nutritional informations per food in order to give a more detailed analysis than the one currently proposed on the meal composed by the patient (only the number and the size of the portions selected are given). Finally, our experience on this kind of application development for HoloLens in a medical context has also allowed us to develop a prototype of needle inserting assistance during hemodialysis sessions [64] or another one to assist surgeons during trocar placement [65].

Acknowledgements Authors would like to thank Arthur Jacquin, student in DUT Informatique Graphique at IUT de Clermont-Ferrand, France (site Le Puy-en-Velay) for the software design. This project is funded by a french national grant: PEPS INSIS CNRS "Sciences de l'Ingénierie pour la Santé pour accompagner des projets translationnels", 2017, project AVACM (Assistance Visuelle Augmentée lors de Consultations Médicales).

\section{References}

1. O. K. Appadoo, H. Rositi, S. Valarier, M.-C. Ombret, E. Gadea-Deschamps, C. BarretGrimault, and C. Lohou, "Multimedia contents design for a mixed reality experience with a HoloLens headset, on the occasion of the digitalization of human nutrition sessions," in review, 2020.

2. S. Hercberg, P. Galan, P. Preziosi, S. Bertrais, L. Mennen, D. Malvy, A.-M. Roussel, A. Favier, and S. Briançon, "The SU.VI.MAX Study: A Randomized, PlaceboControlled Trial of the Health Effects of Antioxidant Vitamins and Minerals," JAMA Internal Medicine, vol. 164, pp. 2335-2342, 112004.

3. S. Hercberg, M. Deheeger, and P. Preziosi, Portions alimentaires : Manuel photos pour l'estimation des quantités. Éditions Polytechnica, 2002.

4. C. Barret-Grimault, M.-C. Ombret, O. K. Appadoo, H. Rositi, S. Valarier, E. Privat, I. Benmabrouk, V. Haas, V. Rousset, S. Verret, C. Lohou, and E. Gadea, "Innovons 
en ETP grâce au casque HoloLens en chirurgie bariatrique !," in Société d'éducation thérapeutique européenne (SETE), $7^{e}$ édition, (Toulouse), May 2019.

5. "HTC Vive headset," 2016.

6. "Oculus Rift," 2016.

7. J. J. LaViola, Jr., "A discussion of cybersickness in virtual environments," SIGCHI Bull., vol. 32, pp. 47-56, January 2000.

8. C. Cruz-Neira, D. J. Sandin, T. A. DeFanti, R. V. Kenyon, and J. C. Hart, "The CAVE: Audio visual experience automatic virtual environment," Commun. ACM, vol. 35, pp. 64-72, June 1992.

9. R. T. Azuma, "A survey of augmented reality," Presence: Teleoper. Virtual Environ., vol. 6, pp. 355-385, August 1997.

10. "Epson Moverio," 2017.

11. "Google Glass," 2013.

12. "Mira augmented reality," 2017.

13. "Meta vision," 2019.

14. J. Watson, M. Firman, A. Monszpart, and G. J. Brostow, "Footprints and free space from a single color image," 2020.

15. Subfigure $2 \mathrm{~A}$ reference, "AS\#04 - mixed reality" by artfulspark and is licensed under CC BY-NC-ND 2.0.

16. Subfigure $2 \mathrm{~B}$ reference, "AR 1 " by cubistscarborough and is licensed under CC BY-NCND 2.0.

17. Subfigure 2C reference, "Augmented Reality Comics@ Ars Electronica Center" by Ars Electronica and is licensed under CC BY-NC-ND 2.0.

18. Subfigure 2D reference, "The view from Cecilia Abadie's driver's seat" by Richard Masoner / Cyclelicious and is licensed under CC BY-SA 2.0.

19. Subfigure $2 \mathrm{E}$ reference, "Drowzee" by Hackley Public Library and is licensed under CC BY 2.0.

20. "Microsoft HoloLens," 2017.

21. Subfigure 3A reference, "File:MicrosoftHoloLensRoomScan.JPG" by Kai Kowalewski and is licensed under CC BY-SA 4.0.

22. "Gaze, Gesture and Voice," 2019.

23. "Kokiri Lab. Project Nourished, a gastronomical virtual reality experience.," 2014.

24. A. Daigneault, "Virtual eating : how virtual reality can make you think you're eating pizza when you're not.," November 2016.

25. F. Rivas, "Students learn the virtual reality of healthy eating and nutrition," April 2017.

26. A. Robertson, "Atkins made a virtual reality game about sugar, and nothing I say can make sense of it," January 2017.

27. J. Auriach, "Régime façon réalité augmentée," September 2017.

28. C.-Y. Ung, M. Menozzi, C. Hartmann, and M. Siegrist, "Innovations in consumer research: The virtual food buffet," Food Quality and Preference, vol. 63, pp. 12 - 17, 2018.

29. M. Ferrer-García and J. Gutiérrez-Maldonado, "The use of virtual reality in the study, assessment, and treatment of body image in eating disorders and nonclinical samples: A review of the literature," Body Image, vol. 9, no. 1, pp. 1 - 11, 2012.

30. S. Persky, M. R. Goldring, S. A. Turner, R. W. Cohen, and W. D. Kistler, "Validity of assessing child feeding with virtual reality," Appetite, vol. 123, pp. $201-207,2018$.

31. R. C. Wolcott, "Virtual reality, sex and chocolate cake: Desire in a post-virtual world," March 2017.

32. C. Perpiñá and M. Roncero, "Similarities and differences between eating disorders and obese patients in a virtual environment for normalizing eating patterns," Comprehensive Psychiatry, vol. 67, pp. $39-45,2016$.

33. M. Meißner, J. Pfeiffer, T. Pfeiffer, and H. Oppewal, "Combining virtual reality and mobile eye tracking to provide a naturalistic experimental environment for shopper research," Journal of Business Research, 2017.

34. M. Z. Bayu, H. Arshad, and N. M. Ali, "Nutritional information visualization using mobile augmented reality technology," Procedia Technology, vol. 11, pp. 396 - 402, 2013. 4th International Conference on Electrical Engineering and Informatics, ICEEI 2013. 
35. D. Igue, "iOS11ARkit : le nouvel OS des iPhone donne la composition des aliments vus par la caméra, en vidéo," July 2017.

36. "Far application," 2017.

37. D. Escárcega-Centeno, A. Hérnandez-Briones, E. Ochoa-Ortiz, and Y. Gutiérrez-Gómez, "Augmented-sugar intake: A mobile application to teach population about sugar sweetened beverages," Procedia Computer Science, vol. 75, pp. 275 - 280, 2015. 2015 International Conference Virtual and Augmented Reality in Education.

38. M. Campbell, "Apple invention uses RFID tags, Apple watch to track food nutrition," May 2017.

39. N. A. ElSayed, B. H. Thomas, K. Marriott, J. Piantadosi, and R. T. Smith, "Situated analytics: Demonstrating immersive analytical tools with augmented reality," Journal of Visual Languages $\&$ Computing, vol. 36, pp. 13 - 23, 2016.

40. J. Menet, "Foodtech : la réalité augmentée au service de l'alimentation," September 2017.

41. O. El-Gayar, P. Timsina, N. Nawar, and W. Eid, "Mobile applications for diabetes selfmanagement: Status and potential," Journal of Diabetes Science and Technology, vol. 7, no. 1, pp. 247-262, 2013. PMID: 23439183.

42. M. E. Rollo, E. J. Aguiar, R. L. Williams, K. Wynne, M. Kriss, R. Callister, and C. E. Collins, "eHealth technologies to support nutrition and physical activity behaviors indiabetes self-management," Diabetes, Metabolic Syndrome and Obesity : Targets and Therapy., vol. 9, pp. 381-390, November 2016.

43. A. Ashman, C. Collins, L. Brown, K. Rae, and M. Rollo, "Evaluation of a mobile phone tool for dietary assessment and to guide nutrition counselling among pregnant women," Journal of Nutrition \& Intermediary Metabolism, vol. 8, p. 90, 2017.

44. M. E. Rollo, T. Bucher, S. P. Smith, and C. E. Collins, "Servar: An augmented reality tool to guide the serving of food," International Journal of Behavioral Nutrition and Physical Activity, vol. 14, p. 65, May 2017.

45. M. Rollo, T. Bucher, S. Smith, and C. Collins, "The effect of an augmented reality aid on error associated with serving food," Journal of Nutrition \& Intermediary Metabolism, vol. 8, p. 90, 062017.

46. C. W. R. University, "HoloAnatomy application," November 2016.

47. A. R. GmbH, "Insight heart application," October 2016.

48. "Vimedix AR ultrasound simulator," August 2017.

49. P. Pratt, M. Ives, G. Lawton, J. Simmons, N. Radev, L. Spyropoulou, and D. Amiras, "Through the HoloLens looking glass: augmented reality for extremity reconstruction surgery using 3D vascular models with perforating vessels," European Radiology Experimental, vol. 2, p. 2, January 2018.

50. Surur, "HoloLens used to plan robotic prostate surgery," January 2017.

51. L. Bremme, "HoloLens : une opération chirurgicale unique au monde en réalité augmentée à Paris," December 2017.

52. F. O'Leary, "Back away from the burger ! Microsoft combats overeating with AR patent," October 2017.

53. "Unity," 2017.

54. "Unreal Engine," 2017.

55. "Blender software," 2018

56. "Microsoft Visual Studio," 2017.

57. "Microsoft HoloToolKit," 2018.

58. C. Lohou, H. Rositi, O. K. Appadoo, S. Valarier, M.-C. Ombret, E. Gadea-Deschamps, and C. Barret-Grimault, "Design of a mixed reality application with a HoloLens headset for a nutrition workshop," in preparation, 2020.

59. "Microsoft HoloLens application," 2016.

60. J. Jang, C. M. Tschabrunn, M. Barkagan, E. Anter, B. Menze, and R. Nezafat, "Threedimensional holographic visualization of high-resolution myocardial scar on hololens," PLOS ONE, vol. 13, pp. 1-14, 102018.

61. I. Kuhlemann, M. Kleemann, P. Jauer, A. Schweikard, and F. Ernst, "Towards X-ray free endovascular interventions - using HoloLens for on-line holographic visualisation," Healthcare Technology Letters, vol. 4, pp. 184-187(3), October 2017. 
62. V. Garcia-Vázquez, F. von Haxthausen, S. Jäckle, C. Schumann, I. Kuhlemann, J. Bouchagiar, A.-C. Höfer, F. Matysiak, G. Hüttmann, J. Peter Goltz, M. Kleemann, F. Ernst, and M. Horn, "Navigation and visualisation with HoloLens in endovascular aortic repair," Innovative Surgical Sciences, vol. 3, pp. 167-177, 102018.

63. H. Liu, E. Auvinet, J. Giles, and F. Rodriguez y Baena, "Augmented reality based navigation for computer assisted hip resurfacing: A proof of concept study," Annals of Biomedical Engineering, vol. 46, pp. 1595-1605, October 2018.

64. C. Lohou, M. Bouiller, and E. Gadea-Deschamps, "Mixed reality experiment for hemodialysis treatment," in Surgetica $9^{\text {th }}$ edition, (Rennes, France), June 2019.

65. C. Lohou, B. Miguel, and K. Azarnoush, "Preliminary experiment of the interactive registration of a trocar for thoracoscopy with HoloLens headset," in $20^{\text {th }}$ International Conference on Imaging Analysis and Processing (ICIAP), (Trento, Italy), September 2019 